\title{
ESTRUCTURA MONOMASTIL EN 132 KV Y 220KV
}

Ing. Esp. Pitrau, Alberto René ${ }^{1}$ Ing. Esp. Sosa, Jorge Omar ${ }^{2}$

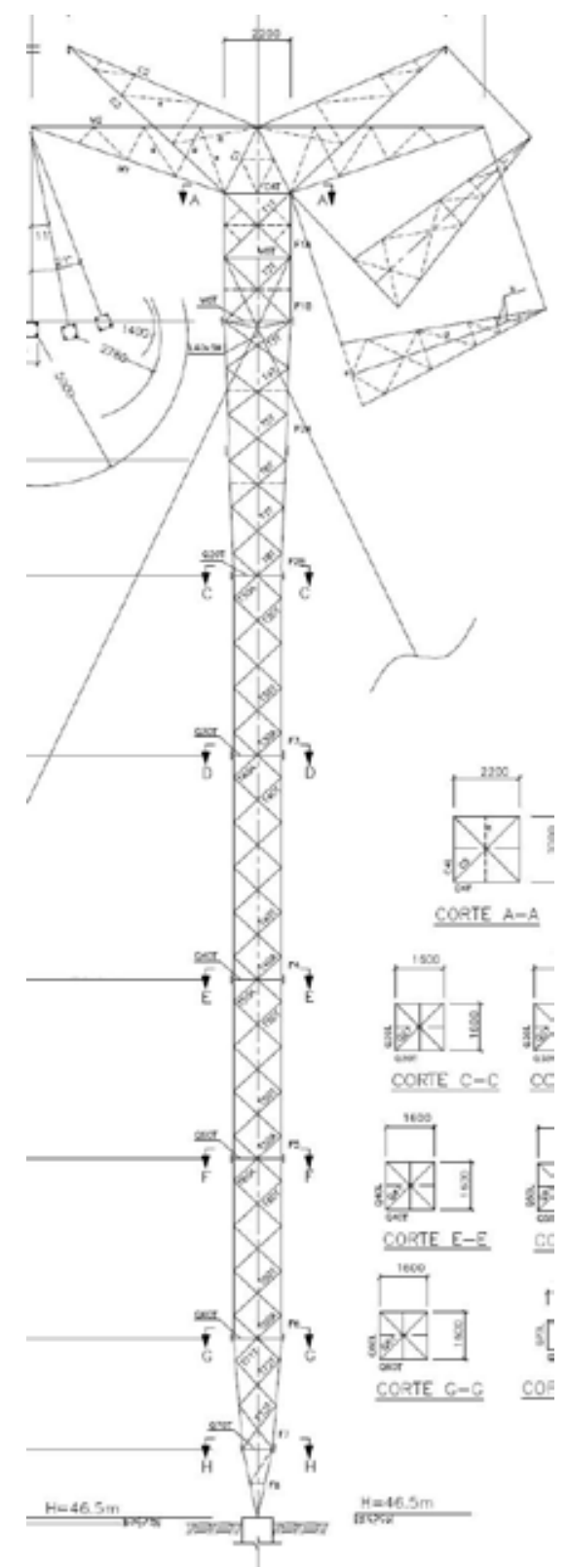

\section{RESUMEN:}

Análisis de las posibilidades de uso de los soportes tipo monomástil en líneas de alta tensión, razones técnicas y económicas para hacerlo. Uso como respuesta rápida ante colapsos de líneas existentes en cualquier tipo de postación.

Palabras clave: Monomástil - arriostrado - colapsos-Líneas de altatensión-estructuras de acero - fundaciones de riendas

\section{ESTRUCTURA MONOMASTIL EN 132 KV Y 220KV}

\section{ANTECEDENTES:}

Las estructuras que estamos acostumbrados a observar y que son utilizadas en alta tensión son básicamente de hormigón armado y acero reticulado. Tal como se ilustra en las imágenes adjuntas. Puede observarse el aspecto monolítico de la estructura triple de $\mathrm{H}^{\circ} \mathrm{A}^{\circ}$ y la complejidad del armado de la torre metálica.

Secretaría de Energía - 25 de Mayo 981 (Corrientes - Cap)

1. Tel: 011-4.501.2333 -albertorenepitrau@fibertel.com.ar

2. Tel: 03624384964 - joromsosa@gmail.com 


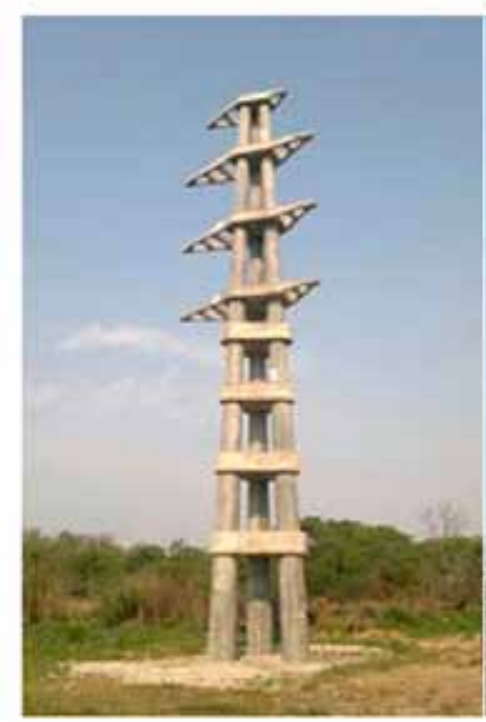

Fig. 1

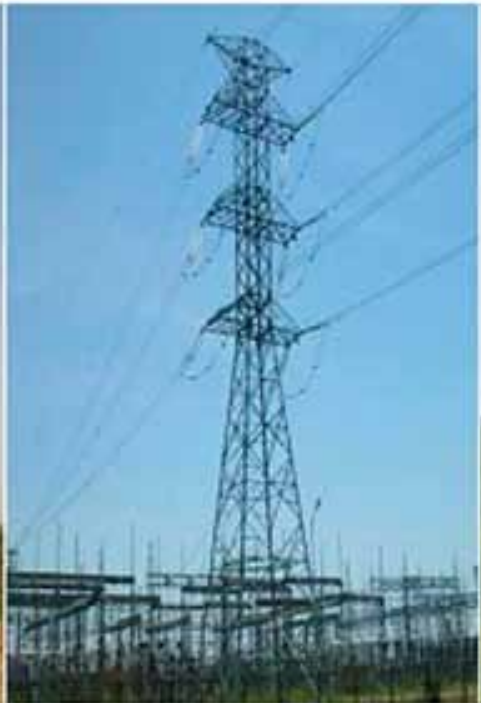

Fig. 2

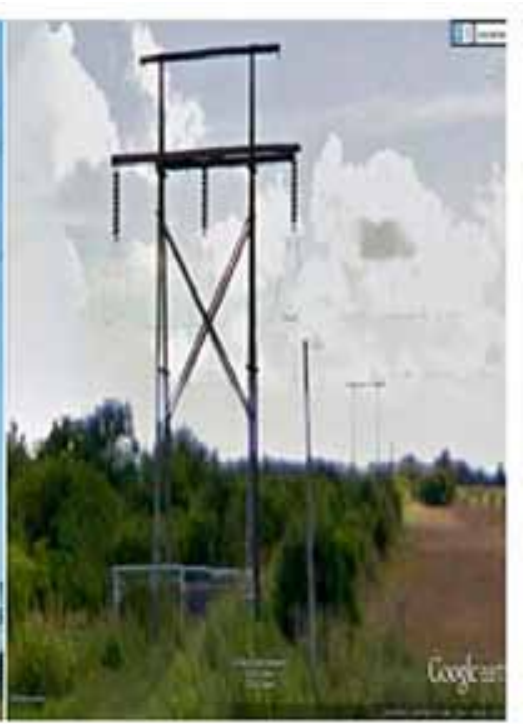

Fig. 3
No son frecuentes las líneas de $132 \mathrm{kv}$ con postación de madera, siendo su uso limitado a estructuras auxiliares en caso de colapso de las originales hasta su reemplazo. Salvo la LAT 132KV Villa Ángela - Charata ejecutada hace 25 años para una emergencia. Se adjunta vista actual donde se observa que esta fuera de servicio.Fig3.

\section{Debilidades y fortalezas de las distintas estructuras}

Columnas de Hormigón armado: Este tipo de estructura, va a tener un peso propio en las suspensiones entre 12 y 14 toneladas, para poder transportar una doble terna de cable de 300/50 $\mathrm{mm}^{2}$ de Al/Ac con cable de OPGW y un cable de acero como cables de guarda, con alturas de $26 \mathrm{mts}$. Desde el punto de vista operativo, su transporte a campo traviesa en las trazas que resultan de vincular dos estaciones transformadoras, implica tener que hacer caminos de accesos y construir o reforzar estructuras existentes de alcantarillas o puentes precarios que en los campos, normalmente no se construyen para el paso de cargas tan pesadas.
Los trabajos viales complementarios, puede constituir en suelos con napas de agua próximas a la superficie, un costo adicional mayor a un $30 \%$ con respecto al total de la obra. Llegado al lugar, se debe hacer el izaje y aplomado de la estructura, dentro de una base de hormigón muy importante, pues si el problema es el suelo, al gran momento de vuelco esperable se requiere una base de hormigón simple o armado de grandes dimensiones, esto implica un transporte al lugar de obra de cantidades de piedra, arena, cemento para $20,30 \mathrm{~m}^{3} \mathrm{o}$ mas, o el uso de mixer para lo cual los caminos deben poder soportar este esfuerzo.

El izaje y aplomado de la columnas y crucetas es complejo por los pesos, esto implica el uso de grúas de gran porte de 30/40 Tn y $30 \mathrm{mts}$ de altura de pluma, que normalmente trabaja en una "isla" de suelo compactado alrededor de la estructura. Los tiempos para el montaje de las estructuras dobles o triples son también muy altos. Lo anterior sumado a que se prevén vanos de 200 a $250 \mathrm{mts}$, implica un costo muy elevado. Ante un eventual colap- 
so pasan largos períodos de tiempo para su reemplazo por el costo que conlleva.

Ventajas que ofrecen las columnas de $\mathrm{H}^{\circ} \mathrm{A}^{\mathbf{o}}$ : se calculan con una carga nominal determinado por el esfuerzo que los vientos y las cargas permanentes que la afectan. Este cálculo se afecta con un

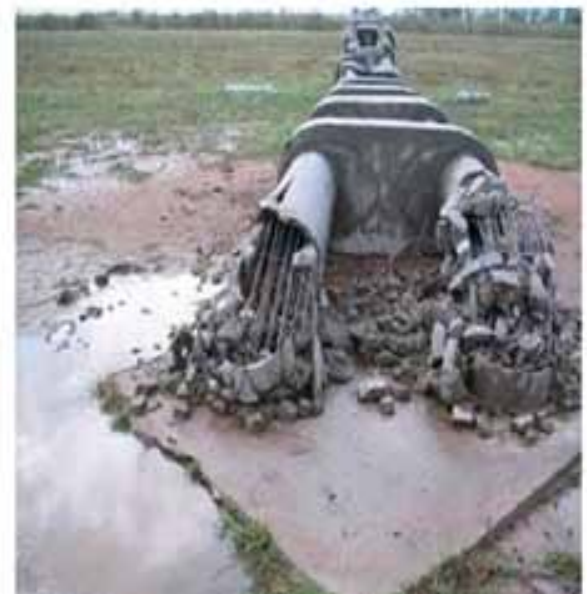

Fig. 4
Estructuras metálicas autosoportadas: Debido a los altos costos que se producen en el montaje de las estructuras de $\mathrm{H}^{\circ} \mathrm{A}^{\circ}$ ( $\mathrm{Si}$ el suelo tiene poca capacidad portante) frecuentemente se utilizan las torres metálicas por la facilidad de manejo de los perfiles, los cuales pueden "tejerse" de manera manual sin el uso de equipos sofisticados o caros, Las bases son mucho menores y actualmente se pueden fabricar externamente las zapatas y parte del fuste, de manera de poder prearmar las bases con hierros descubiertos arriba, calculados para hormigonar con los perfiles de arranque empotrados en las bases (stubs) y de esta manera independientemente de la altura de la napa poder tener un hormigón en la base de alta calidad. La evaluación del uso de soportes de $\mathrm{H}^{\circ} \mathrm{A}^{\circ} \mathrm{o}$ de torres de $\mathrm{H}^{\circ} \mathrm{G}^{\circ}$ va a depender en definitiva de la economía, la torre metálica es más cara que la columna coeficiente de seguridad, lo que la hace excepcionalmente robusta y capaz de resistir vientos máximos superiores a los que pueden aparecer en nuestra zona en periodos muy largos de tiempo. Igualmente caen en tormentas muy severas o tornados como sucedió en varias oportunidades.

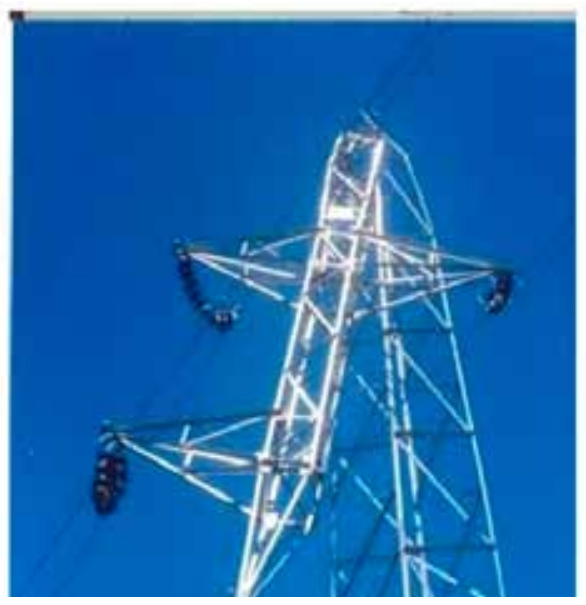

Fig. 5 equivalente, pero no tiene los costos de caminos, alcantarillas y grúas de gran porte, además las torres me permiten optimizar su uso con un vano mayor al de las columna, en una relación 4/3 de máximo, lo que abarata la comparación en un $25 \%$.

Problemas en el uso de torres autosoportadas: Cuando se calculan los esfuerzos, los vientos tomados según las hipótesis de cargas, convertidos a cargas estáticas aplicadas a la estructura no reflejan la realidad según lo que se puede apreciar en los eventos en los que una masa de aire que se desplaza a una determinada velocidad, contiene en su interior ráfagas de mayor velocidad y poca duración, lo que somete a las piezas a esfuerzos no previstos y no poseen una componente elástica que permita absorber el mismo. Una diagonal que falla en una tormenta puede originar la sa- 
lida de servicio de la LAT por colapso de la estructura, a veces con fallas en cascada.

Estructuras de madera: A pesar de no contar habitualmente con líneas de transmisión en estas estructuras, sino que se emplea como auxiliares ante colapsos de las estructuras de hormigón o $\mathrm{H}^{\circ} \mathrm{G}^{\circ}$, la experiencia recogida con los postes de máxima altura que tenemos en el país, no permite tener vanos ni de la mitad de las otras estructuras, soportan menor sección de conductor, poca duración de la madera tratada, requerir un mantenimiento permanente por quema de campos, poca rigidez estructural, etc.

\section{ESTRUCTURAS TIPO MONO- MASTIL: RAZONES PARA SU UTI- LIZACION}

\section{Concepto de estructura ${ }^{(1)}$}

La estructura es la manera de conseguir la máxima resistencia con el minimo material, mediante la utilización más apropiada de las formas y los materiales. Conseguir lo máximo mediante lo minimo. La estructura no consiste en hacer algo más fuerte agregando masa y volumen, sino utilizando menos materiales de la manera más apropiada consiguiendo así la resistencia necesaria. También debe atenderse a las necesidades de transporte de las estructuras, desde la fábrica hasta la obra, en lo referido a los tamaños y pesos involucrados, y cómo comprometen a los equipos de montaje necesarios, asi como los requerimientos de mano de obra especializada (y en esto influye también la ubicación física de la obra).Pensemos en las cargas que se presentan, la forma de la estructura, los materiales de la estructura, y evaluemos las alternativas posibles; de todas ellas debe elegirse la mejor.

La intuición de equilibrio y estabilidad que aun poseen los profanos, es un conjunto de conocimientos desordenados; éstos, sistema- tizados y ordenados, unidos al conocimiento de los materiales disponibles, y la manera como se generan y canalizan las fuerzas, ayudan a un buen diseño. El "diseño estructural" es la etapa previa al "cálculo estructural". Para el primero no se requieren excesivos y profundos conocimientos matemáticos.

(1) (extraído de publicación de la UNLP del Ing. Bernal (UNNE))

El incremento de la extensión de líneas de transmisión de alta tensión, aumenta la probabilidad de ser impactadas por tormentas de gran intensidad, teniendo estas en su interior corrientes descendentes, tornados, etc. Poniendo en evidencia nuevos fenómenos no observados hasta el momento.

Los soportes que se calculan de acuerdo a las hipótesis de carga según las normas vigentes, y que son impactados por tormentas, caen en algunos casos en cascada produciendo importantes pérdidas materiales por la reposición de lo destruido, por la falta de energía vendida, pérdidas de producción por tener en muchos casos trabajos electrodependientes, costos sociales por la pérdida de calidad de vida, etc.

De las observaciones en las torres de hierro reticulado de tipo autosoportado (las clásicas del tipo de las de la Figura 2) ante la presencia de esfuerzos dinámicos, (debido a ráfagas viento de corta duración < a 5 segundos) que se presentan en tormentas importantes, se origina el colapso de las estructuras, ya que no pueden absorber la energía mecánica del impacto del viento que se distribuye en el soporte produciendo flexión lateral en algunos componentes. Es obvio que siempre se puede ir sobredimensionando la torre para que soporte vientos extremos lo cual sería antieconómico y no proporcionaría una seguridad al 100\% nunca, 
ya que tornados que se forman en las grandes tormentas igualmente las derribarían.

¿Cómo resolver o atenuar los efectos de los esfuerzos dinámicos, que producen deformaciones $y$ destruyen los soportes?

La respuesta la podemos encontrar en la flexibilidad, por ejemplo en los soportes de madera, cuando no están carcomidos, por su flexibilidad los soportes de madera tienen un excelente comportamiento a las fuerzas variables y con un empotramiento razonable pueden soportar vientos que hacen colapsar estructuras de hormigón o hierro galvanizado. (Como antecedente: cuando cayeron en la LEAT de $500 \mathrm{KV}$

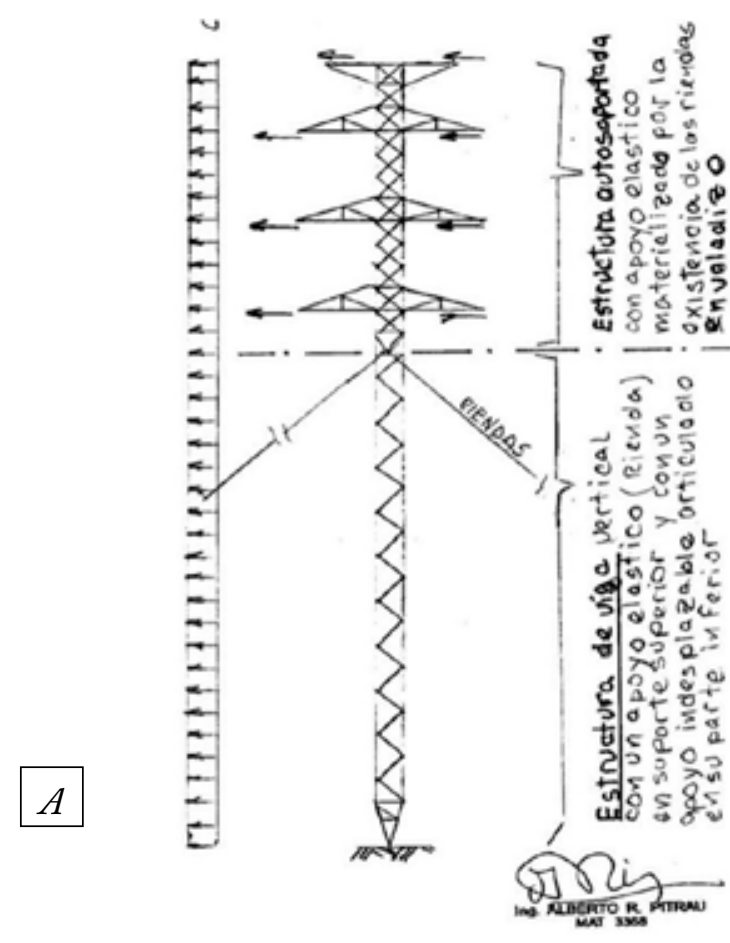

las estructuras de $\mathrm{H}^{\circ} \mathrm{G}^{\circ}$, los de madera auxiliar de $132 \mathrm{kv}$, que reemplazaban los soportes de $\mathrm{H}^{\circ} \mathrm{G}^{\circ}$ caídos en eventos anteriores, sufrieron pocos daños relativos).

\section{¿Qué configuración podemos encon-}

trar, para que soporte cargas de gran intensidad y poca duración, con la capacidad de transporte de potencia necesaria?

La respuesta puede encontrarse en la manera de aprovechar los mástiles de las estructuras tipo cross rope, en las cuales los mástiles reciben una carga de compresión, mientras que los esfuerzos de tracción son absorbidos por la riendas convenientemente fijas al terreno mediante anclajes de hormigón de diferentes formas según el estudio de suelos, o soluciones más rápidas donde puedan efectuarse como las helicodes tipo change o similar.

En la siguiente bibliografía (2 Rev. Int.

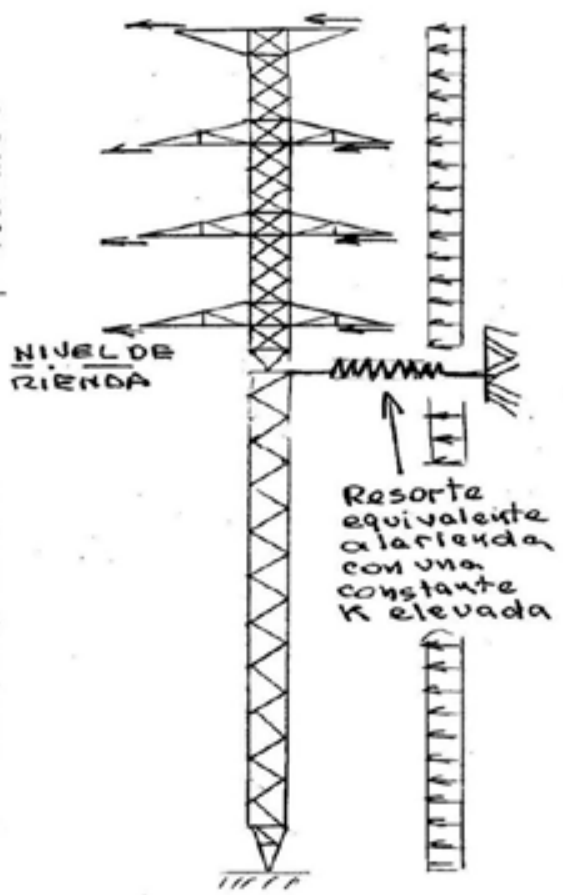

De Desastres Naturales, Accidentes e infraestructura civil. Vol 6 (1)) varios autores han presentado análisis para esfuerzos dinámicos de soportes, donde según se desprende el funcionamiento del sistema viga columna es no lineal, siendo esta no linea- 
lidad resultado de la interacción de grandes cargas axiales normalmente cercanas a las del pandeo y los desplazamientos laterales que ocurren en condiciones normales de trabajo. Se analiza en el trabajo (2) los esfuerzos dinámicos extremos que ocurren en ráfagas de vientos intensos, vibraciones por vorticidad, presencia de masas rotantes desbalanceadas, etc. Esto lo resume el Ing. Alberto Pitrau en el siguiente análisis:

a) Existen dos comportamientos distintos de la estructura (Fig. A) según se la considere por encima o debajo de la ménsula de fijación de las riendas.

La estructura arriostrada en conjunto columna y riendas constituyen globalmente una estructura autosoportada, mucho más flexible que una estructura autosoportada tradicional, por el comportamiento de las riendas. Las riendas constituyen un resorte equivalente con una elevada constante elástica $k=$ Carga aplicada/Deformación.

La rienda como toda pieza que trabaja a la tracción posee una reserva adicional, dada por el diagrama característico de los aceros.

\section{ENSAYO DE TRACCIÓN}

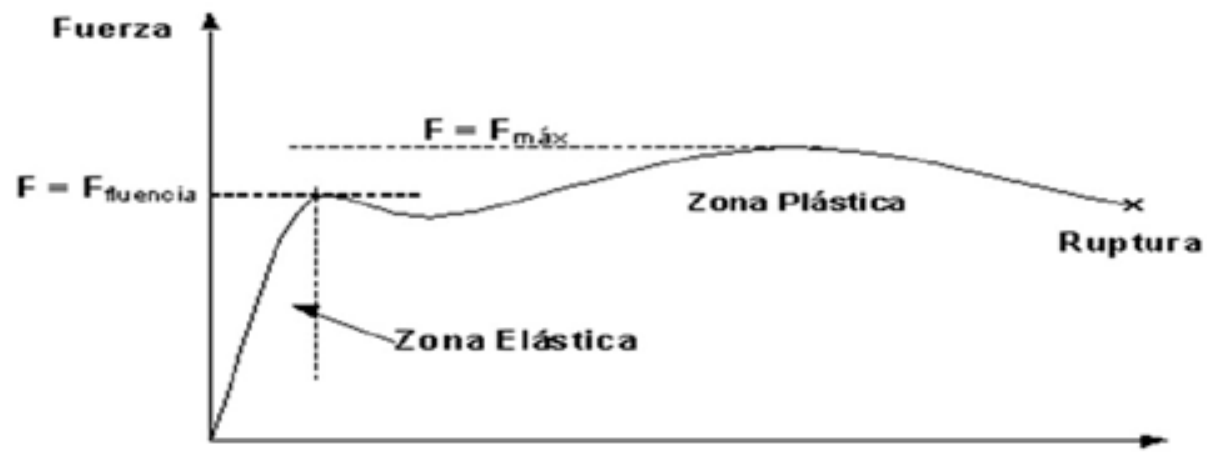

Desplazamiento

$f x 1,50$, la reserva adicional estaría dada por esta relación. Si consideramos un resorte con un peso que se encuentra en equilibrio e incrementamos la carga, se produce un desplazamiento y oscilación, quedando en equilibrio en otra posición, siendo su comportamiento igual al de una rienda con carga.

El diagrama de vientos en corte $\mathrm{v}$, la $\mathrm{v}$ es igula a 0 en correspondencia con el terreno debido a la rugosidad del mismo. Esto lo podemos resumir como la suma de dos efectos:

a) Un viento de velocidad moderada que se puede considerar constante a lo largo de la traza. Tomando como valor medio sobre un período de diez minutos

b) Un viento en forma de ráfagas (valor máximo instantáneo) actúa con distintas magnitudes en un TRAMO ACOTADO de la línea. A la suma de $a+b$ es lo que normalmente denominamos ráfaga de vientos y esta puede ser de tal magnitud que puede sobrepasar la resistencia de algunas estructuras. El sistema cable + aisladores es deformable y hace que la carga que estos transmiten a los estructuras es la integral sobre un sistema acotado de vanos (entre 2 y 4 ) y produciendo sobre la misma una magnitud de carga muy 
importante. Si simultáneamente sobre una de estas estructuras actua (a) + (b) (se denomina FACTOR DE RAFAGA a la relación entre Ráfaga instantánea/Valor medio del viento), esto puede llevar a las estructuras en cuestión al colapso. Este efecto será más letal cuanto más rígido sea el comportamiento de la torre o columna de $\mathrm{H}^{\circ} \mathrm{A}^{\circ}$.

Es decir de acuerdo a este criterio podemos poner en orden de riesgo:

1) Postes de Hormigón Armado

2) Torres autosoportadas que son masrígidas que los monomastiles (por lo dicho más arriba) y poseen una mayor área expuesta a la ráfaga de viento

3) Torres monomástil, son más flexibles que los postes de hormigón (Rotura frágil) y que las torres autosoportadas empotradas en correspondencia con el terreno.

Soporte monomástil como auxiliar para colapsos de cualquier LAT

Una ventaja tan importante como su resistencia a esfuerzos dinámicos, es su facilidad de montaje, dado que puede instalarse como los mástiles de las estructuras tipo crossrope, erigiendo el mástil girando su rotula de contacto con la pieza empotrada en la base de hormigón. La industrialización de las estructuras, permite una gran seguridad en el trabajo, dado que se llevan pre armados al campo y se posicionan con la facilidad comentada, es decir montaje sin riesgos y de manera rápida, permitiendo un control exhaustivo de los torques de la bulonerìa y detalles de su armado.

Versatilidad en la configuración como reemplazo para cualquier Soporte
Teniendo en cuenta las diferentes configuraciones de líneas existentes, el empleo de estructuras monomástil en configuración doble terna coplanar vertical, permitirá su empleo como estructura de reemplazo en caso de colapso para cualquier sistema existente, ya que las bases y placas de anclaje premoldeadas se pueden transportar con facilidad y colocarlas en suelos de difícil acceso y la configuración de la torre puede armarse colocando o eliminando las ménsulas que no han de emplearse. Siendo esta ventaja comparativa fundamental al momento de tomar una decisión sobre el sistema a emplear. Unificando modelos se obtienen las mayores ventajas al momento de encarar un colapso del sistema de transmisión.

Este tipo de estructuras podrán reemplazar en plazos muy cortos, estructuras de hormigón o auto soportadas metálicas que hayan colapsado.

Aspecto económico de la líneas de alta tensión con soportes monomàstil

Peso de la estructura: podemos agregar que el peso calculado para una doble terna monomàstil es inferior en casi un tercio a una torre autosoportada de la misma prestación.

Resistencia a los esfuerzos dinámicos: Del análisis anterior surge claramente que esta resistencia en estructuras arriendadas, al tener los esfuerzos de tracción soportadas por las riendas de manera elástica, permite una prestación muy superior a las autosoportadas del mismo costo.

Bases de hormigón versus riendas con anclaje: Dependiendo del suelo considerado podemos tener una correspondencia entre 4 bases de hormigón contra 1 sola y cuatro 
riendas con anclaje de placas de $\mathrm{H}^{\circ} \mathrm{A}^{\circ}$ o troncos de cono con gancho de anclaje.

Vanos: Se prevé con la estructura tipo monomàstil un vano un $20 \%$ superior al de la estructura autosoportada, lo cual implica un $20 \%$ menos de aisladores, morseterìa, bases, montaje, etc.

Facilidad de reparación: por lo comentado al inicio tanto la reparación como el cambio completo de la estructuras es posible en muy poco tiempo, en suelos como el de nuestra provincia considero la mejor solución posible.
Conclusiones: La economía de esta estructura es evidente, un $10 \%$ o un $20 \%$ menos por $\mathrm{km}$ de LAT. A lo debemos añadir una mejor resistencia a los esfuerzos dinámicos que son responsables de los colapsos más conocidos. (Dado que a vientos medidos uniformes, los cálculos verificaban que se soportaban sin embargo las estructuras caìan). La evolución de las LEAT de $500 \mathrm{KV}$ nos muestran una evolución desde las autosoportadas, a las del tipo delta, a las crossrope por razones de economía y seguridad que es perfectamente trasladable a nuestra propuesta para Lineas de 132 o $220 \mathrm{KV}$. 